九州大学学術情報リポジトリ

Kyushu University Institutional Repository

\title{
A Study on the Generation of Field-Aligned Current in the Magnetosphere
}

Itonaga, Masahiro

Faculty of Education, Yamaguchi University

Yoshikawa, Akimasa

Faculty of Sciences, Kyushu University

Yumoto, Ki yohumi

Faculty of Sciences, Kyushu University

Fujita, Shigeru

Meteorologlcal College

他

https://doi.org/10.5109/1546805

出版情報: 九州大学大学院理学研究院紀要：Series D, Earth and planetary sciences. 31 (1)，pp. 19, 2000-12-25. Faculty of Science, Kyushu University バージョン :

権利関係 : 
Mem. Fac. Sci., Kyushu Univ., Ser. D, Earth \& Planet. Sci., Vol. XXXI, No. 1, pp. 1-9, text-figs. 1-3, December 25, 2000

\title{
A Study on the Generation of Field-Aligned Current in the Magnetosphere
}

\author{
M. ITONAGA*, A.YOSHIKAWA, K.YUMOTO, S.FujiTA**, and H.NAKATA***
}

\begin{abstract}
The field-aligned current (FAC) is a key element in the magnetosphere-ionosphere coupling. A wave equation describing the generation of $\mathrm{FAC}$ in the magnetosphere has been derived by ITONAGA et al. (2000). This equation presents a deep insight into the coupling of the FAC with the inertial and diamagnetic currents associated with magnetosonic disturbances via the effects of inhomogeneous medium and curvilinear magnetic field line. It is assumed in the derivation of the wave equation that the flow velocity disappears in the unperturbed state. Under the condition of $u$ « $V$, where $u$ is the magnitude of unperturbed flow velocity and $V$ the propagation speed of MHD disturbance, this assumption is valid. The generation of quasi-oscillatory current wedge with a time scale of 1 to 2 min is discussed on the basis of the wave equation.
\end{abstract}

\section{Introduction}

The field-aligned current (FAC) is a key element in the magnetosphere-ionosphere coupling and the elucidation of its generation mechanism is one of the most important subjects in magnetospheric physics. In the magnetohydrodynamic (MHD) limit, the FAC is not constrained by dynamical conditions describing motion along the magnetic field line. That is, since the electron parallel mobility is assumed to be infinite, the FAC is simply determined by a current closure requirement that the divergence of current density vanishes everywhere. For most of theoretical works on the FAC, such a requirement becomes the starting point along with the momentum equation (HaSegawa and Sato, 1979; SATO and IjImA, 1979; SATO, 1982; VASYliunas, 1984; ITONAGA et al., 2000). The divergence of the perpendicular current is divided apparently into a part associated with the parallel component of vorticity of the plasma flow and other parts proportional to the inertial and diamagnetic currents flowing perpendicularly to the magnetic field. HASEGAWA and SATO (1979) put forward such an expression describing the generation of FACs during magnetospheric substorms. The solar wind energy injected to the magnetotail is converted into plasma flow energy. When the plasma flow meets the inner magnetosphere, a viscous interaction occurs and creates time-increasing plasma flow vorticities. They found that the interaction produces FACs upward and downward above the ionosphere in the pre-midnight and post-midnight sectors, respectively. However, HASEGAWA and SATO's expression is valid only when the vorticity is everywhere parallel to the magnetic field and such an assumption is generally quite unrealistic, as pointed out by

\footnotetext{
Manuscript received August 8, 2000 ; accepted October 16, 2000.

${ }^{*}$ Faculty of Education, Yamaguchi University, Yamaguchi 753-8513, Japan

** Meteorological College, Kashiwa 277-0852, Japan

${ }^{* * *}$ Solar-Terrestrial Environment Laboratory, Nagoya University, Toyokawa 442-8507, Japan
} 
VASYLIUNAS (1984). Further, the separation of the FAC into vorticity dependent and independent parts in their expression is not unique.

Recently, ITONAGA et al. (2000) derived a wave equation of a new kind describing the generation of FAC in the magnetosphere. This equation presents a deep insight into the coupling of the FAC with the inertial and diamagnetic currents associated with magnetosonic disturbances via the effects of inhomogeneous medium and curvilinear magnetic field line. In the derivation of the wave equation, they assumed that the flow velocity disappears in the unperturbed state. However, in general, there exist plasma convections and flows in the magnetosphere. Then, one may doubt whether such an assumption is valid. In the present paper we will examine the validity of the assumption. We will also show that the wave equation derived by ITONAGA et al. (2000) has the advantage of a linearized version of the expression put forward by HASEGAWA and SATO (1979). Further, on the basis of the wave equation, we will discuss the formation of a quasi-oscillatory current wedge at the braking point of high-speed ion flows pointed out by SHIOKAWA et al. (1997, 1998).

\section{Validity of Zero Unperturbed Flow Velocity}

ITONAGA et al. (2000) assumed that the flow velocity disappears in the unperturbed state. Considering the magnetosphere to be filled with a warm magnetized plasma, we examine the validity of this assumption. In the unperturbed state,

$$
\begin{gathered}
\nabla \cdot(\rho \mathbf{u})=0, \\
\rho(\mathbf{u} \cdot \nabla) \mathbf{u}=-\nabla p+\mathbf{j} \times \mathbf{B}, \\
\nabla \times \mathbf{E}=\mathbf{0}, \\
\nabla \times \mathbf{B}=\mu_{0} \mathbf{j}, \\
\mathbf{E}+\mathbf{u} \times \mathbf{B}=\mathbf{0}
\end{gathered}
$$

hold in the magnetosphere, where $\rho, \mathbf{u}, \mathbf{E}, \mathbf{B}, \mathbf{j}$ and $p$ represent the unperturbed plasma density, flow velocity, electric field, magnetic field, current density and plasma pressure, respectively. Further, $\mu_{0}$ is the permeability of vacuum. Then, linearized equations become

$$
\begin{gathered}
\frac{\partial \delta \rho}{\partial t}+\nabla \cdot(\rho \delta \mathbf{u})+\nabla \cdot(\delta \rho \mathbf{u})=0, \\
\rho \frac{\partial \delta \mathbf{u}}{\partial t}+\rho(\mathbf{u} \cdot \nabla) \delta \mathbf{u}+\rho(\delta \mathbf{u} \cdot \nabla) \mathbf{u}+\delta \rho(\mathbf{u} \cdot \nabla) \mathbf{u}=-\nabla \delta p+\mathbf{j} \times \delta \mathbf{B}+\delta \mathbf{j} \times \mathbf{B}, \\
\nabla \times \delta \mathbf{E}=-\frac{\partial \delta \mathbf{B}}{\partial t}, \\
\nabla \times \delta \mathbf{B}=\mu_{0} \delta \mathbf{j}, \\
\delta \mathbf{E}+\delta \mathbf{u} \times \mathbf{B}+\mathbf{u} \times \delta \mathbf{B}=\mathbf{0}
\end{gathered}
$$

for the perturbation quantities which are denoted with the prefix $\delta$.

If the third term in the lefthand side of Eq. (6) can be neglected, the first and second terms must 
have magnitudes of the same order and so $|\delta \rho| / \rho \sim \delta u / V$, where $\delta u$ is the magnitude of $\delta \mathbf{u}$ and $V$ the propagation speed of MHD disturbance. When $V_{\mathrm{A}}$ and $V_{\mathrm{S}}$ represent the Alfvén and sound speeds, respectvely, $V$ is of the order of $V_{\mathrm{A}}$ and/or $V_{\mathrm{s}}$. Let $u$ denote the magnitude of $\mathbf{u}$. Then, since

$$
\left|\frac{\nabla \cdot(\delta \rho \mathbf{u})}{\nabla \cdot(\rho \delta \mathbf{u})}\right| \sim \frac{|\delta \rho|}{\rho} \frac{u}{\delta u} \sim \frac{u}{V}
$$

$u \ll V$ is the condition to be capable of neglecting the third term.

Under the condition of $u \ll V$, only the first term survives in the lefthand side of Eq. (7), because

$$
\left|\frac{\rho(\mathbf{u} \cdot \nabla) \delta \mathbf{u}}{\rho \frac{\partial \delta \mathbf{u}}{\partial t}}\right| \sim \frac{u}{V} \ll 1, \quad\left|\frac{\rho(\delta \mathbf{u} \cdot \nabla) \mathbf{u}}{\rho \frac{\partial \delta \mathbf{u}}{\partial t}}\right| \lesssim \frac{u}{V} \ll 1, \quad\left|\frac{\delta \rho(\mathbf{u} \cdot \nabla) \mathbf{u}}{\rho \frac{\partial \delta \mathbf{u}}{\partial t}}\right| \lesssim\left(\frac{u}{V}\right)^{2} \ll 1,
$$

where it is assumed that the characteristic length in spatial variation of the perturbation quantity is smaller than that of the unperturbed one. From Eq. (2) we have

$$
\mathbf{j}_{\perp}=\frac{1}{B^{2}} \mathbf{B} \times[\rho(\mathbf{u} \cdot \nabla) \mathbf{u}]+\frac{1}{B^{2}} \mathbf{B} \times \nabla p,
$$

where the subscript $\perp$ stands for a vector component perpendicular to $\mathbf{B}$ and $B$ is the magnitude of B. Letting $\mathbf{j}_{\perp}^{(\mathrm{i})}$ and $\mathbf{j}_{\perp}^{\text {(d) }}$ denote the first and second terms in the righthand side of Eq. (11), respectively, we divide $\mathbf{B}$ into three parts, $\mathbf{B}^{(\mathrm{e})}, \mathbf{B}^{(\mathrm{i})}$ and $\mathbf{B}^{(\mathrm{d})}$, where $\nabla \times \mathbf{B}^{(\mathrm{e})}=\mathbf{0},\left(\nabla \times \mathbf{B}^{(\mathrm{i})}\right)_{\perp}=\mu_{0} \mathbf{j}_{\perp}^{(\mathrm{i})}$ and $\left(\nabla \times \mathbf{B}^{(\mathrm{d})}\right)_{\perp}=\mu_{0} \mathbf{j}_{\perp}^{(\mathrm{d})}$. That is, $\mathbf{B}^{(\mathrm{e})}$ is produced by any other unperturbed current than the magnetospheric one, while $\mathbf{B}^{(\mathrm{i})}\left(\mathbf{B}^{(\mathrm{d})}\right)$ arises from $\left.\mathbf{j}_{\perp}^{(\mathrm{i})} \mathbf{j}_{\perp}^{(\mathrm{d})}\right)$ and its associated FAC. Then, since

$$
\left|\frac{\rho(\mathbf{u} \cdot \nabla) \mathbf{u}}{\nabla p}\right| \sim \frac{\rho u^{2}}{p} \sim\left(\frac{u}{V_{s}}\right)^{2},
$$

$\mathbf{j}_{\perp}^{\text {(i) }}$ can be neglected as compared with $\mathbf{j}_{\perp}^{\text {(d) }}$ under the condition of $u \ll V_{\mathrm{S}}$, meaning that the magnitude of $\mathbf{B}^{(\mathrm{i})}$ is much smaller than that of $\mathbf{B}^{(\mathrm{d})}$. Further, when $\nabla_{\perp} p$ is parallel to $\nabla_{\perp} p_{B}$, where $p_{B}$ $=B^{2} / 2 \mu_{0}$ is the unperturbed magnetic pressure, the FAC due to the divergence of $\mathbf{j}_{\perp}^{\left({ }_{\perp}\right)}$ vanishes (or $\nabla \cdot \mathbf{j}_{\perp}^{\text {(d) }}=0$ ). Thus, under the condition of $u \ll V$ and $\nabla_{\perp} p \| \nabla_{\perp} p_{B}$, Eq. (7) leads to

$$
\rho \frac{\partial \delta \mathbf{u}}{\partial t}=-\nabla \delta p+\mathbf{j}_{\perp}^{(\mathrm{d})} \times \delta \mathbf{B}+\delta \mathbf{j}_{\perp} \times \mathbf{B}
$$

which has been used in ITONAGA et al. (2000). Further, $\mathbf{B} \simeq \mathbf{B}^{(\mathrm{e})}+\mathbf{B}^{(\mathrm{d})}$ can be represented in terms of Euler potentials (STERN, 1967; SOUTHWOOD and KIVELSON, 1991), although the divergence of $\mathbf{j}_{\perp}^{(\mathrm{i})}$ may yield the FAC in the unperturbed state.

If the third term in the lefthand side of Eq. (10) can be neglected, the first and second terms must have magnitudes of the same order and so $\delta E \sim \delta u B$, where $\delta E$ is the magnitude of $\delta \mathbf{E}$. On the other hand, $\delta E \sim V \delta B$ from Eq. (8), where $\delta B$ is the magnitude of $\delta \mathbf{B}$. Then, we take $\delta B / B \sim$ $\delta u / V$. Since

$$
\left|\frac{\mathbf{u} \times \delta \mathbf{B}}{\delta \mathbf{u} \times \mathbf{B}}\right| \sim \frac{u}{\delta u} \frac{\delta B}{B} \sim \frac{u}{V}
$$

$u \ll V$ is also the condition to be capable of neglecting the third term. Thus, under the condition of $u \ll V$, Eq. (10) results in

$$
\delta \mathbf{E}_{\perp}+\delta \mathbf{u}_{\perp} \times \mathbf{B}=\mathbf{0}
$$


In general, there exist plasma convections and flows (or $\mathbf{u} \neq \mathbf{0}$ ) in the magnetosphere. However, if $u \ll V\left(u \ll V_{\mathrm{A}}\right.$ and $\left.u \ll V_{\mathrm{S}}\right)$, the assumption that the flow velocity $\mathbf{u}$ disappears in the unperturbed state will be valid. Since the convective speed due to the Earth rotation is only $3 \mathrm{~km} / \mathrm{s}$ or so even at $L=6$, the above condition will be sure to hold in the region of dipolar magnetic field relatively near the Earth. In the distant magnetosphere, on the other hand, it is thought that the flow speed is much larger than the convective speed. Then, the validity of the assumption must be carefully examined in such a region. In the cold plasma limit, the condition of $u \ll V$ (or $u \ll V_{\mathrm{A}}$ ) means that $\mathbf{j}$ little flows, that is, $\nabla \times \mathbf{B} \simeq \mathbf{0}$.

\section{Advantage of Wave Equation}

On the basis of Eqs. (8), (9), (12) and (13), ITONAGA et al. (2000) derived a wave equation

$$
\begin{aligned}
& \frac{1}{V_{\mathrm{A}}^{2}} \frac{\partial^{2} \delta j \|}{\partial t^{2}}-\frac{1}{V_{\mathrm{A}}^{2}} \frac{\partial}{\partial s}\left[V_{\mathrm{A}}^{2} B \frac{\partial}{\partial s}\left(\frac{\delta j \|}{B}\right)\right]=-\frac{1}{V_{\mathrm{A}}^{2}} \frac{\partial}{\partial s}\left(\nabla_{\perp} V_{\mathrm{A}}^{2} \cdot \delta \mathbf{j}_{\perp}^{(i)}\right) \\
& +\frac{1}{V_{\mathrm{A}}^{2}}\left[\nabla^{2}\left(V_{\mathrm{A}}^{2} \delta \mathbf{j}_{\perp}^{(\mathrm{i})}\right)\right]_{1} \\
& -\frac{1}{V_{\mathrm{A}}^{2}} \frac{\partial}{\partial s}\left(\frac{V_{\mathrm{A}}^{2}}{p_{B}} \nabla_{\perp} p_{B} \cdot \delta \mathbf{j}_{\perp}^{(\mathrm{d})}\right) \\
& -\frac{1}{V_{\mathrm{A}}^{2}} \frac{\partial}{\partial s}\left[\frac{V_{\mathrm{A}}^{2}}{2 p_{B}} \nabla_{\perp}\left(\delta p_{B}-\delta p\right) \cdot \mathbf{j}_{\perp}^{(\mathrm{d})}\right]
\end{aligned}
$$

describing the generation of FAC in the magnetosphere, where $\delta j_{\|}$is the perturbation FAC and

$$
\begin{aligned}
\delta \mathbf{j}_{\perp}^{(\mathrm{i})} & =\frac{1}{B^{2}} \mathbf{B} \times\left(\rho \frac{\partial \delta \mathbf{u}_{\perp}}{\partial t}\right), \\
\delta \mathbf{j}_{\perp}^{(\mathrm{d})} & =\frac{1}{B^{2}} \mathbf{B} \times \nabla_{\perp} \delta p, \\
\mathbf{j}_{\perp}^{(\mathrm{d})} & =\frac{1}{B^{2}} \mathbf{B} \times \nabla_{\perp} p .
\end{aligned}
$$

The variable $s$ is a distance measured along the magnetic field line and increases in the direction of B. The subscript $\|$ stands for a scalar component parallel to B. Further, $\delta p_{B}=B \delta B_{\|} / \mu_{0}$ is the perturbation magnetic pressure.

Equation (14) has four source terms. The first and second terms in the righthand side of Eq. (14) indicate the effects of inhomogeneous Alfvén speed and curvilinear magnetic field line, respectively. The second term vanishes in the case of straight field line or $R_{\mathrm{c}}=\infty$, where $R_{\mathrm{c}}$ denotes the radius of curvature of the magnetic field line. When there exist the inhomogeneity of the Alfvén speed and the curvature of the magnetic field line, the perturbation FAC $\delta j_{\|}$is produced by the perpendicular perturbation inertial current $\delta \mathbf{j}_{\perp}^{(\mathrm{i})}$. On the other hand, the third term shows that $\delta j_{\|}$ is caused by the perpendicular perturbation diamagnetic current $\delta \mathbf{j}_{\perp}^{(\mathrm{d})}$ in case of the existence of inhomogeneity of the unperturbed magnetic pressure. The generation of FAC via the third term was investigated by SOUTHWOOD and KIVELSON (1991) in a quite different form. Further, when the perpendicular diamagnetic current flows in the unperturbed state, the perturbations of the magnetic and plasma pressures bring about $\delta j_{\|}$, as seen from the fourth term. Figure 1 is a schematic diagram for the generation of perturbation FAC. Here, $\delta j_{\|}^{(\text {i) }}$ and $\delta j_{\|}^{(\text {d) }}$ denote the

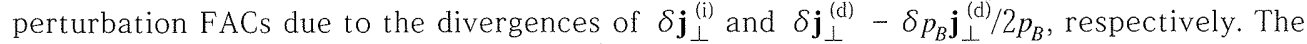
perturbation FAC $\delta j_{\|}$consists of $\delta j_{\|}^{(\mathrm{i})}$ and $\delta j_{\|}^{(\mathrm{d})}$. 


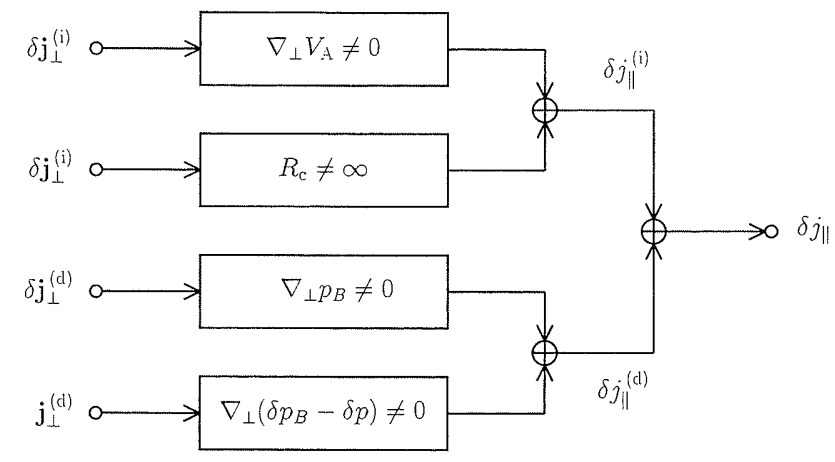

Fig. 1. Schematic diagram for the generation of perturbation FAC. When the Alfven speed is inhomogeneous $\left(\nabla_{\perp} V_{\mathrm{A}} \neq 0\right)$ and the magnetic field line is curvilinear $\left(R_{\mathrm{C}} \neq \infty\right)$, the perpendicular perturbation inertial current $\delta \mathbf{j}_{\perp}^{(i)}$ produces the perturbation FAC $\delta j_{\|}^{(i)}$. When the unperturbed magnetic pressure is inhomogeneous $\left(\nabla_{\perp} p_{B} \neq 0\right.$ ), the perpendicular perturbation diamagnetic current $\delta \mathbf{j}_{\perp}{ }_{\perp}^{(d)}$ yields the perturbation FAC $\delta j_{\|}$(d). When the perpendicular diamagnetic current $\mathbf{j}_{\perp}{ }^{(\mathrm{d})}$ flows in the unperturbed state, the perturbations of the magnetic and plasma pressures $\left(\nabla_{\perp}\left(\delta p_{B}-\bar{\delta} p\right) \neq 0\right)$ also bring about the perturbation FAC $\delta j_{\|}^{(\mathrm{d})}$. The perturbation FAC $\delta j_{\|}$consists of $\delta j_{\|}^{(\mathrm{i})}$ and $\delta j_{\|}^{(\text {d) }}$ due to the perpendicular perturbation inertial and diamagnetic currents.

Now, let us consider a linearized version of the expression put forward by HASEGAWA and SATO (1979). Since

$$
\delta \mathbf{j}_{\perp}=\delta \mathbf{j}_{\perp}^{(\mathrm{i})}+\delta \mathbf{j}_{\perp}^{(\mathrm{d})}-\frac{1}{2 p_{B}} \delta p_{B} \mathbf{j}_{\perp}^{(\mathrm{d})}
$$

from Eq. (12), we have

$$
B \frac{\partial}{\partial s}\left(\frac{\delta j \|}{B}\right)=-\nabla \cdot \delta \mathbf{j}_{\perp}^{(\mathrm{i})}-\nabla \cdot\left(\delta \mathbf{j}_{\perp}^{(\mathrm{dd})}-\frac{1}{2 p_{B}} \delta p_{B} \mathbf{j}_{\perp}^{\text {(d) }}\right)
$$

from $\nabla \cdot \delta \mathbf{j}=0$. The divergence of $\delta \mathbf{j}_{\perp}^{(\mathrm{i})}$ has a complex form. After some manipulations we can obtain

$$
\nabla \cdot \delta \mathbf{j}_{\perp}^{(\mathrm{i})}=-\rho \frac{\partial}{\partial t}\left(\frac{\delta \Omega_{\|}}{B}\right)+\text { other terms, }
$$

where $\delta \Omega_{\|}=(\nabla \times \delta \mathbf{u})_{\|}$is the parallel component of vorticity of the perturbation plasma flow. Other terms in the righthand side of Eq. (19) depend on $\delta \mathbf{j}_{\perp}^{(\mathrm{i})}, \delta \mathbf{j}_{\perp}^{(\mathrm{d})}$ and $\mathbf{j}_{\perp}^{(\mathrm{d})}$, and so their physical meanings

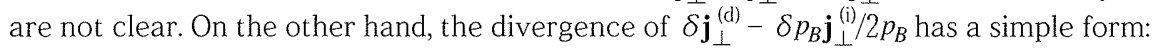

$$
\nabla \cdot\left(\delta \mathbf{j}_{\perp}^{(\mathrm{d})}-\frac{1}{2 p_{B}} \delta p_{B} \mathbf{j}_{\perp}^{(\mathrm{d})}\right)=-\frac{1}{p_{B}} \nabla_{\perp} p_{B} \cdot \delta \mathbf{j}_{\perp}^{(\mathrm{d})}-\frac{1}{2 p_{B}} \nabla_{\perp}\left(\delta p_{B}-\delta p\right) \cdot \mathbf{j}_{\perp}^{(\mathrm{d})} .
$$

The linearized version is given by Eqs. (18), (19) and (20).

From Eq. (20) we take

$$
\frac{\delta j \|}{B}=\int_{0}^{(\mathrm{d})}\left[\frac{1}{p_{B}} \nabla_{\perp} p_{B} \cdot \delta \mathbf{j}_{\perp}^{(\mathrm{d})}+\frac{1}{2 p_{B}} \nabla_{\perp}\left(\delta p_{B}-\delta p\right) \cdot \mathbf{j}_{\perp}^{(\mathrm{d})}\right] \frac{d s^{\prime}}{B},
$$

where it is assumed that $\delta j_{\|}^{(\mathrm{d})}$ vanishes at the magnetic equator $(s=0)$. If the distributions of $\delta p$ and $\delta p_{B}$ are known, $\delta j_{\|}^{(\mathrm{d})}$ can be calculated directly using Eq. (21). Then, as far as $\delta j_{\|}^{(\mathrm{d})}$ is concerned, the use of Eq. (14) is not so much advantageous. However, since the first and second terms in the righthand side of Eq. (14) have simple forms and their physical meanings are clear, Eq. (14) is much more advantageous in considering $\delta j_{\|}^{(i)}$ than the expression directly derived from Eq. (19). 


\section{Discussion}

Equation (14) is a linear formula and so it cannot be applied to such a highly nonlinear process as the formation of substorm current wedge with a time scale of about 30 to $60 \mathrm{~min}$ in the magnetosphere. The nonlinear process is nowadays studied directly with numerical simulations (e.g., TANAKA, 1995). However, it is thought that some small-amplitude quasi-oscillatory currents with time scales of about 1 to 2 min superposed on the large-amplitude slowly-varying wedge current cause Pi 2 pulsations (LeSTER et al., 1983; BAUMJOHANN and GLASSMEIER, 1984; ITONAGA and Yuмoto, 1998). The cross-tail portion of the quasi-oscillatory current wedge generates a fast magnetosonic wave. During its passage within the magnetosphere the magnetosonic wave excites shear Alfvén waves owing to the effects of inhomogeneous medium and curvilinear magnetic field line. Since the formation of quasi-oscillatory current wedge and the associated process have not been yet simulated numerically, the use of Eq. (14) will be of great advantage to their theoretical studies.

Recently, SHIOKAwA et al. (1997) studied braking mechanisms of high-speed ion flows in the near-Earth central plasma sheet and suggested that the high-speed flows are braked at a clear boundary between the regions of dipolar and tail-like magnetic fields in the plasma sheet. The boundary corresponds to the inner edge of the neutral sheet. SHIOKAwA et al. (1998) have pointed out that the dawnward inertial currents caused by the braking of ion flow and the pileup of magnetic flux at the braking point can be a cause of the wedge current during the initial stage of the substorm expansion phase. Then, we assume that the quasi-oscillatory current wedge as well as the substorm current wedge is formed at the braking point (or the inner edge of the neutral sheet). The slowly-varying wedge current can be regarded as an unperturbed current. However, it should be noted that the existence of large-amplitude unperturbed FAC harms the effectiveness of Eq. (12). Then, the application of Eq. (14) based on Eq. (12) to the formation of quasi-oscillatory current wedge will be limited to the the initial stage of the substorm expansion phase.

Introducing Euler potentials $\Phi$ and $\phi$ (STERN, 1967; SOUTHWOOD and KIVELSON, 1991), we represent the ambient magnetic field with $\mathbf{B}=\nabla \Phi \times \nabla \phi$. We can require $\nabla \Phi \cdot \nabla \phi=0$ without loss of generality. The Euler potentials $\Phi$ and $\phi$ are constant along each magnetic field line and label the meridional and longitudinal directions, respectively. Further, we introduce an orthogonal coordinate system $(\Phi, \phi, \chi)$ based on the Euler potentials, where $\chi$ varies along each magnetic field line and increases in the direction of $\mathbf{B}$. Figure 2 illustrates the orthogonal coordinate system. The scale factors of $\Phi, \phi$ and $\chi$ are denoted by $h_{\Phi}, h_{\phi}$ and $h_{\chi}$, respectively.

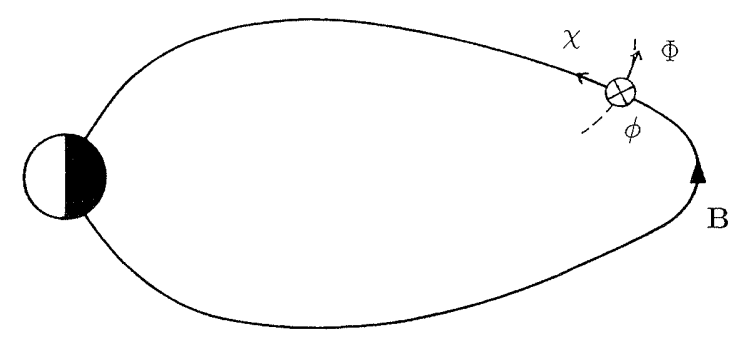

Fig. 2. Orthogonal coordinate system $(\Phi, \phi, \chi)$. The Euler potentials $\Phi$ and $\phi$, which are constant along each magnetic field line, label the meridional and longitudinal directions, repectively, while $\chi$ varies along each field line and increases in the direction of the ambient magnetic field $\mathbf{B}$. 
Around the inner edge of the neutral sheet, the magnitude of the second term in the righthand side of Eq. (14) would be at most of the order of that of the first one, because the ambient magnetic field is approximately dipolar. If $V_{\mathrm{A}}$ has a sharp gradient in the $\Phi$ direction, the first term will dominate over the second one (ITONAGA et al., 2000). Further, it would be reasonably expected that the unperturbed diamagnetic current $\mathbf{j}_{\perp}^{(\mathrm{d})}$ is dawnward there as well as $\partial V_{A}^{2} / \partial \Phi<0$ and $\partial p_{B} / \partial \Phi<0$. It should be noted that the first and second terms have the same sign because of $\partial V_{A}^{2} / \partial \Phi<0$ (ITONAGA et al., 2000). Unless $V_{\mathrm{A}}$ has a significant gradient in the $\phi$ direction,

$$
-\frac{1}{V_{\mathrm{A}}^{2}} \frac{\partial}{\partial s}\left(\nabla_{\perp} V_{\mathrm{A}}^{2} \cdot \delta \mathbf{j}_{\perp}^{(i)}\right) \simeq-\frac{1}{V_{\mathrm{A}}^{2}} \frac{1}{h_{\triangleright}} \frac{\partial V_{A}^{2}}{\partial \Phi} \frac{1}{h_{\chi}} \frac{\partial \delta_{\infty}^{(i)}}{\partial \chi}
$$

holds (ITONAGA et al., 2000). Then, the dawnward inertial current caused by the braking of highspeed ion flow will hardly produce the perturbation FAC directly. However, the braking would bring about an increase of the plasma pressure $(\delta p>0)$ around the inner edge of the neutral sheet and the resultant plasma pressure gradient would accelerate the surrounding plasma. Then, $\partial \delta u_{\phi} / \partial \mathrm{t}$ $<0$ because of $\partial \delta p / \partial \phi>0$ in the pre-midnight sector and $\partial \delta u_{\phi} / \partial \mathrm{t}>0$ because of $\partial \delta p / \partial \phi<0$ in the post-midnight sector. This acceleration results in $\delta \mathbf{j}_{\perp}^{(\mathrm{i})}$ such as $\delta j_{\Phi}^{(\mathrm{i})}>0$ and $\delta j_{\Phi}^{(\mathrm{i})}<0$ in the preand post-midnight sectors, respectively (see Eq. (15)). Since the gradient of $V_{\mathrm{A}}$ lies primarily in the $\Phi$ direction, the $\Phi$ component of $\delta \mathbf{j}_{\perp}^{(\mathrm{i})}$ can produce $\delta j_{\|}$effectively (see Eq. (22)). Further, $\nabla_{\perp} V_{A}^{2} \cdot \delta \mathbf{j}_{\perp}^{(\mathrm{i})}$ $\left\langle 0\right.$ and $\left.\nabla_{\perp} V_{\mathrm{A}}^{2} \cdot \delta \mathbf{j}_{\perp}^{(\mathrm{i})}\right\rangle 0$ in the the pre- and post-midnight sectors, respectively, because of $\partial V_{\mathrm{A}}^{2} / \delta \Phi$ $<0$. Hence, the initial sense in flow direction of $\delta j_{\|}$due to the first term agrees with the sense of FAC of the substorm current wedge. Figure 3 shows the generation of perturbation FAC due to the braking of high-speed ion flow.

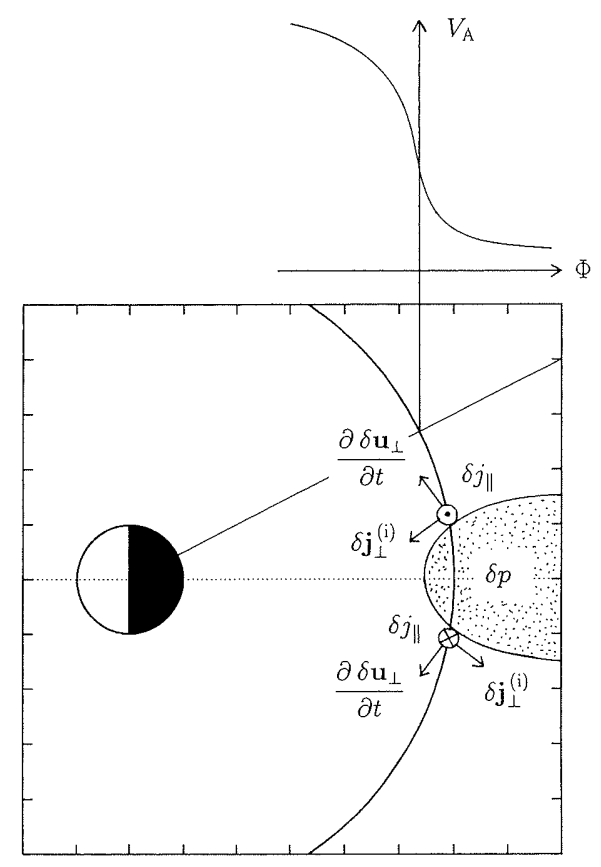

Fig. 3. Generation of perturbation FAC due to the braking of high-speed ion flow. The braking brings about an increase of the plasma pressure $(\delta p>0)$ around the inner edge of the neutral sheet and the resultant plasma pressure gradient accelerates the surrounding plasma. The acceleration of plasma results in the perpendicular perturbation inertial current $\delta \mathbf{j}_{\perp}^{(i)}$ and this current produces the perturbation FAC $\delta j_{\|}$ via the effects of inhomogeneous Alfvén speed and curvilinear magnetic field line. 
The third and fourth terms in the righthand side of Eq. (14) might also play important roles in the generation of perturbation FAC around the inner edge of the neutral sheet. The gradient of $\delta p$ causes $\delta \mathbf{j}_{\perp}^{(\mathrm{d})}$ such as $\delta j_{\Phi}^{(\mathrm{d})}<0$ and $\delta j_{\Phi}^{(\mathrm{d})}>0$ in the pre- and post-midnight sectors, respectively (see Eq. (16)). Since the gradient of $p_{B}$ also lies primarily in the $\Phi$ direction and $\partial p_{B} / \partial \Phi\left\langle 0, \nabla_{\perp} p_{B} \cdot \delta \mathbf{j}_{\perp}^{\text {(d) }}\right\rangle 0$ and $\nabla_{\perp} p_{B} \cdot \delta \mathbf{j}_{\perp}^{(\mathrm{d})}<0$ in the pre- and post-midnight sectors, respectively. Then, the initial sense in flow direction of $\delta j_{\|}$due to the third term is opposite to the sense of FAC of the substorm current wedge. If the disturbance due to the braking of high-speed ion flow has a diamagnetic property, the increase of plasma pressure will yield the decrease of magnetic pressure. Then, $\partial \delta p_{B} / \partial \phi<0$ and $\partial \delta p_{B} / \partial \phi>0$ in the pre- and post-midnight sectors, respectively. Since $\mathbf{j}_{\perp}^{(\mathrm{d})}$ is dawnward, $\nabla_{\perp}\left(\delta p_{B}-\right.$ $\delta p) \cdot \mathbf{j}_{\perp}^{(\mathrm{d})}<0$ and $\nabla_{\perp}\left(\delta p_{B}-\delta p\right) \mathbf{j}_{\perp}^{(\mathrm{d})}>0$ in the pre- and post-midnight sectors, respectively. Hence, the initial sense in flow direction of $\delta j_{\|}$due to the fourth term is in agreement with the sense of FAC of the substorm current wedge. Thus, the third and fourth terms produce the perturbation FACs with opposite initial senses in flow direction. In the case of $\beta \sim 1$, however, these terms have magnitudes of the same order (ITONAGA et al., 2000), meaning that these terms cancel out each other. Then, only the first and second terms could contribute to the generation of perturbation FAC.

The perturbation FACs caused by the braking of high-speed ion flow propagate along magnetic field lines and reflect at the polar ionosphere. As a result, quasi-periodic field line oscillations of shear Alfvén waves occur at auroral latitudes in the pre- and post-midnight sectors. The sense in flow direction of the perturbation FAC associated with the field line oscillation in the pre-midnight sector is opposite to that in the post-midnight sector. These perturbation FACs with opposite senses are closed via the ionospheric and cross-tail perturbation currents. Thus, the quasioscillatory current wedge with a time scale of 1 to 2 min will be generated and it will yield Pi 2 pulsations. In the present study it has been shown that the FAC of Pi 2 pulsation initially has the same flow direction as that of the substorm current wedge. This is also supported by observations (e.g., BAumjohann and Glassmeier, 1984).

\section{Acknowledgments}

This work was partly supported by the Grant-in-Aid for Scientific Research (C), No. 09640532, from The Ministry of Education, Science and Culture.

\section{References}

Baumjohann, W. and K.-H. Glassmeier (1984): The transient response mechanism and Pi2 pulsations at substorm onset - Review and outlook. Planet. Space Sci., 32, 1361-1370.

Hasegawa, A. and T. Sato (1979): Generation of field aligned current during substorm. in Dynamics of the Magnetosphere, ed. by S.-I. AKASOFu, pp. 529-542, D. Reidel, Hingham, Mass.

ITONAGA, M. and K. Yumoto (1998): ULF waves and the ground magnetic field. J. Geophys. Res., 103, 9285-9291.

Itronaga, M., A. Yoshikawa, and S. FujiTa (2000): A wave equation describing the generation of fieldaligned current in the magnetosphere. Earth Planets Space, 52, 503-507.

Lester, M., W. J. Hughes, and H. Jinger (1983): Polarization pattern of Pi 2 magnetic pulsations and the substorm current wedge. J. Geophys. Res., 88, 7958-7966.

Sato, T. (1982): Auroral physics. in Magnetospheric Plasma Physics, ed. by A. NISHIDA, pp. 197-243, D. Reidel, Hingham, Mass.

Sato, T. and T. Iıjma (1979): Primary sources of large-scale Birkeland currents. Space Sci. Rev., 24, 
347-366.

Shiokawa, K., W. Baumjohann, and G. HaEREndel (1997): Braking of high-speed flows in the nearEarth tail. Geophys. Res. Lett., 24, 1179-1182.

Shiokawa, K., W. Baumjohann, G. Haerendel, G. Paschmann, J. F. Fennel, E. Fris-Christensen, H. LüHR, G. D. ReEves, C. T. Russell, P. R. Sutcliffe, and K. TAKahashi (1998): High-speed ion flow, substorm current wedge, and multiple Pi 2 pulsations. J. Geophys. Res., 103, 4491-4507.

SouTHWOOD, D. J. and M. G. KIVELSON (1991): An approximate description of field-aligned currents in a planetary magnetic field. J. Geophys. Res., 96, 67-75.

STERn, D. (1967): Geomagnetic Euler potentials. J. Geophys. Res., 72, 3995-4005.

TANAKA, T. (1995): Generation mechanisms for magnetosphere-ionosphere current systems deduced from a three-dimensional MHD simulation of the solar wind-magnetosphere-ionosphere coupling processes. J. Geophys. Res., 100, 12,057-12,074.

VASYLIUNAS, V. M. (1984): Fundamentals of current description. in Magnetospheric Currents, ed. by T. A. POTEMRA, pp. 63-66, AGU, Washington, D.C. 